\title{
Lessons of the month 2: Meningococcal epiglottitis and connective tissue disease associated with $\mathrm{C} 2$ deficiency
}

\author{
Authors: Darija Čubelić, ${ }^{A}$ Dylan James Mac Lochlainn, ${ }^{B}$ Elizabeth Bateman ${ }^{C}$ and Siraj A Misbah ${ }^{D}$
}

\begin{abstract}
Case presentation
A 68-year-old woman was referred for immunological investigation following an episode of meningococcal epiglottitis with associated septicaemia. Several years previously, she had been diagnosed with undifferentiated connective tissue disease. On investigation, alternative pathway complement function was normal; however, classical pathway complement activation was reduced. C1q, C3 and C4 levels were all measured and found to be within their respective normal ranges, but $\mathrm{C} 2$ levels were low. Sequencing of the C2 gene was subsequently performed, confirming a diagnosis of type 1 C2 deficiency (C2D).

\section{Discussion}

C2D is usually hereditary and inherited in an autosomal recessive manner. C2D is often asymptomatic, however, some patients suffer from infections with encapsulated bacteria and/or autoimmune diseases, particularly systemic lupus erythematosus. Recognition of complement pathway deficiency is important due to the predisposition to severe and/or recurrent infections by encapsulated bacteria. Immunisations have the potential to reduce both mortality and morbidity not only for the patient but also for any affected relatives.
\end{abstract}

KEYWORDS: meningococcal disease, connective tissue disease, complement deficiency, C2 deficiency

DOI: 10.7861/clinmed.2021-0529

\section{Case presentation}

A 68-year-old woman was referred for investigation following a complex intensive care admission for meningococcal epiglottitis. Blood cultures had grown Neiserria meningitidis (serogroup Y). Apart from one episode of left periorbital cellulitis, she had no other significant history of infections.

Authors: ${ }^{A}$ consultant immunologist, University Hospital Centre

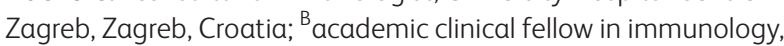

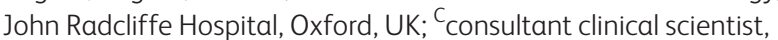
Churchill Hospital, Oxford, UK; ${ }^{\text {D }}$ consultant immunologist, John Radcliffe Hospital, Oxford, UK
Several years previously, she had been diagnosed with undifferentiated connective tissue disease. Her presenting features included a purpuric rash over her lower limbs, shortness of breath and a non-productive cough. She had no sicca symptoms, Raynaud's phenomenon, fatigue or joint pain. She was found to have a positive antinuclear antibody screen with positive anti-Ro antibodies, a positive rheumatoid factor, negative anti-cyclic citrullinated peptide antibodies and polyclonal hypergammaglobulinaemia. Left lower zone changes had been noted on a chest $X$-ray, and subsequent high-resolution computed tomography (HRCT) showed left lower zone ground-glass opacification with interlobular septal thickening but no established fibrosis. The appearances were reported as being consistent with non-specific interstitial pneumonia. She was initially treated with hydroxychloroquine, however, this medication was not tolerated due to associated nausea and was discontinued after 3 months. As her symptoms had fully resolved, no further treatment was deemed necessary at that stage.

There was no additional relevant past medical history, and the patient was not on any regular medication. There was no significant family medical history, specifically of recurrent or severe infections or autoimmune diseases. Physical examination at the time of her review was unremarkable, as were urinalysis and initial blood tests, including a full blood count, urea and electrolyte profile, and liver function tests.

Given the history of both meningococcal infection and connective tissue disease, complement studies were performed. While alternative pathway complement function was normal, classical pathway complement activation was less than $15 \%$ of the normal control on two separate occasions suggesting a deficiency in early components of the classical complement pathway. C1q, C3, and C4 levels were all measured and found to be within their respective normal ranges, however, $\mathrm{C} 2$ levels were found to be low at $2.7 \mathrm{mg} / \mathrm{L}$ (normal range 14-25). Sequencing of the $\mathrm{C} 2$ gene was subsequently performed, and the patient was found to be homozygous for a known deletion (c.841_849+19del28), confirming a diagnosis of type 1 C2 deficiency (C2D).

While the patient had not suffered any further infections or deterioration in her symptoms to date, her pulmonary function tests had been declining on serial measurements. A repeat HRCT did not show any evidence of disease progression, thus, it was decided that immunosuppression was not indicated at present. Nevertheless, she remains under review with her respiratory physician and will continue to have regular lung function tests. The 
patient received immunisations against encapsulated organisms (including meningococcus, pneumococcus and Haemophilus influenza) and her first-degree relatives were offered screening for classical pathway complement deficiency.

\section{Discussion}

C2 is a component of the classical complement pathway, which also includes $\mathrm{C} 1 \mathrm{q}, \mathrm{C} 1 \mathrm{r}, \mathrm{C} 1 \mathrm{~s}$ and C4. C2D is usually hereditary and is inherited in an autosomal recessive manner. In the more common type $1 \mathrm{C} 2 \mathrm{D}$, there is an absence of translated protein, usually caused by a 28 base pair deletion resulting in the generation of a premature termination codon; this allele is present in $1 \%$ of healthy western Europeans. 'The rarer type 2 C2D is due to a failure in the secretion of the translated protein. ${ }^{2}$

C2D is frequently asymptomatic, however, some patients suffer from infections with encapsulated bacteria and/or autoimmune diseases, particularly systemic lupus erythematosus. ${ }^{3} \mathrm{~A}$ high rate of cardiovascular disease has also been reported. ${ }^{3}$ One large case series showed that over half of C2D patients experienced infections, which were pneumococcal rather than meningococcal in the majority of cases. ${ }^{1}$ However, meningococcal infection was associated with virtually all cases of terminal complement pathway defects and is the predominant type of infection when complement deficiencies are considered as a group. ${ }^{1}$

The recognition of a complement pathway deficiency is important, as although the deficient proteins cannot be replaced, the predisposition to severe and/or recurrent infections by encapsulated bacteria can be addressed. Immunisations and antibiotic prophylaxis, where appropriate, have the potential to reduce both mortality and morbidity not only for the patient but also affected relatives, who may not have experienced infections. Although C2D is autosomal recessive in inheritance, the high frequency of the pathogenic allele in the western European population should be kept in mind. ${ }^{1}$

The present UK joint specialist societies guideline on acute meningitis and meningococcal sepsis for adult patients recommends 'appropriate immunological investigations' for all adult patients with two or more episodes of meningococcal or pneumococcal meningitis and for all patients who have a family history of more than one episode of meningococcal disease. ${ }^{4}$ Investigation is not recommended for those with a single episode of meningitis or meningococcal disease unless there is 'some other indication'. Although complement deficiency is mentioned as a predisposing condition in the guideline, the additional indications for its investigation are not. ${ }^{4}$
The case we have presented is a practical illustration of an appropriate indication for further immunological investigation when only one episode of meningococcal disease has been documented: the co-existence of a connective tissue disease. A further noteworthy point in this case is that the patient's age at the time of meningococcal infection is atypical. The incidence of meningococcal disease with respect to age is bimodally distributed with one peak in children less than 5 years of age and a second peak in adolescence and early adulthood. ${ }^{4}$

The predisposition to autoimmune disease as well as serious infections that can occur with classical complement component deficiencies can give rise to a treatment dilemma, as the need may arise for immunosuppression, which would further predispose to infection. Consequently, careful communication between specialty teams and with the patient in such situations is necessary, weighing up the potential risks and benefits of foregoing or proceeding with immunosuppressive treatment, while optimising prevention of infection through vaccination and antibiotic prophylaxis.

\section{Conflicts of interest}

Dylan James Mac Lochlainn is supported by an academic clinical fellowship from the National Institute for Health Research.

\section{References}

1 El Sissy C, Rosain J, Vieira-Martins P et al. Clinical and genetic spectrum of a large cohort with total and sub-total complement deficiencies. Front Immunol 2019;10:1936.

2 Johnson CA, Densen P, Hurford RK Jr et al. Type I human complement C2 deficiency. A 28-base pair gene deletion causes skipping of exon 6 during RNA splicing. J Biol Chem 1992;267:9347-53.

3 Jönsson G, Truedsson L, Sturfelt G et al. Hereditary C2 deficiency in Sweden: frequent occurrence of invasive infection, atherosclerosis, and rheumatic disease. Medicine (Baltimore) 2005;84:23-34.

4 McGill F, Heyderman RS, Michael BD et al. The UK joint specialist societies guideline on the diagnosis and management of acute meningitis and meningococcal sepsis in immunocompetent adults. J Infect 2016;72:405-38.

Address for correspondence: Dr Siraj A Misbah, Department of Clinical Immunology, Oxford University Hospitals NHS Foundation Trust, John Radcliffe Hospital, Headley Way, Headington, Oxford OX3 9DU, UK. Email: Siraj.Misbah@ouh.nhs.uk 